The Steady Propagation of an Air Finger into a Rectangular Tube

De Lozar, Alberto and Juel, Anne and Hazel, Andrew 2008

Manchester Institute for Mathematical Sciences

School of Mathematics

The University of Manchester

\footnotetext{
Reports available from: http://eprints.maths.manchester.ac.uk/

And by contacting: The MIMS Secretary

School of Mathematics

The University of Manchester

Manchester, M13 9PL, UK
} 


\title{
The steady propagation of an air finger into a rectangular tube
}

\author{
ALBERTO DE LÓZAR, ANNE JUEL AND \\ ANDREW L. HAZEL \\ Manchester Centre for Nonlinear Dynamics and School of Mathematics, \\ University of Manchester, M13 9PL, UK
}

(Received 14 February 2008 and in revised form 7 July 2008)

The steady propagation of an air finger into a fluid-filled tube of uniform rectangular cross-section is investigated. This paper is primarily focused on the influence of the aspect ratio, $\alpha$, on the flow properties, but the effects of a transverse gravitational field are also considered. The three-dimensional interfacial problem is solved numerically using the object-oriented multi-physics finite-element library oomph-lib and the results agree with our previous experimental results (de Lózar et al. Phys. Rev. Lett. vol. 99, 2007, article 234501) to within the $\pm 1 \%$ experimental error.

At a fixed capillary number $\mathrm{Ca}$ (ratio of viscous to surface-tension forces) the pressure drops across the finger tip and relative finger widths decrease with increasing $\alpha$. The dependence of the wet fraction $m$ (the relative quantity of liquid that remains on the tube walls after the propagation of the finger) is more complicated: $m$ decreases with increasing $\alpha$ for low $\mathrm{Ca}$ but it increases with $\alpha$ at high $\mathrm{Ca}$. Our results also indicate that the system is approximately quasi-two-dimensional for $\alpha \geqslant 8$, when we obtain quantitative agreement with McLean \& Saffman's two-dimensional model for the relative finger width as a function of the governing parameter $1 / B=12 \alpha^{2} \mathrm{Ca}$. The action of gravity causes an increase in the pressure drops, finger widths and wet fractions at fixed capillary number. In particular, when the Bond number (ratio of gravitational to surface-tension forces) is greater than one the finger lifts off the bottom wall of the tube leading to dramatic increases in the finger width and wet fraction at a given $\mathrm{Ca}$.

For $\alpha \geqslant 3$ a previously unobserved flow regime has been identified in which a small recirculation flow is situated in front of the finger tip, shielding it from any contaminants in the flow. In addition, for $\alpha \gtrsim 2$ the capillary number, $C a_{c}$, above which global recirculation flows disappear has been observed to follow the simple empirical law: $C a_{c}^{2 / 3} \alpha=1.21$.

\section{Introduction}

The displacement of one fluid by a second, immiscible, fluid is a phenomenon present in many applications including enhanced oil recovery, flow in porous media, thin-film coating and the biomechanics of the lung. The canonical model problem concerns a finger, or long bubble, of air that is driven through a tube of uniform cross-section, initially filled by a wetting fluid.

The majority of previous studies of this model problem have considered two particular tube geometries: (i) a circular tube (Bretherton 1961), and (ii) a Hele-Shaw cell, a rectangular tube whose width is much greater than its depth so that the system 
is approximately two-dimensional (Saffman \& Taylor 1958). In many applications, however, the tubes are neither circular nor quasi-two-dimensional. In particular, many pharmaceutical and food processing applications require an understanding of interfacial flows at microfluidic scales (Baroud \& Willaime 2004). In microfluidic devices the manufactured microchannels are usually of rectangular cross-section, but with widths comparable to their depths.

Motivated by these applications, recent studies have investigated air-finger propagation in polygonal tubes, see the recent review by Ajaev \& Homsy (2006). The tubes are usually of rectangular cross-section, which can be characterized by a single aspect ratio $\alpha=w / b$, defined to be the ratio of the tube cross-section width $w$ to its depth $b$. If the aspect ratio is large, the Hele-Shaw cell geometry is recovered, in which the displacement of viscous fluid by a propagating air finger is known as the Saffman-Taylor problem. The Saffman-Taylor problem is an archetype of pattern-forming instabilities and has been extensively studied in the literature, see the review of Homsy (1987). Fewer studies have been conducted at low aspect ratios, but for $1 \leqslant \alpha \leqslant 2$ steady air-finger propagation in fluid-filled rectangular tubes has been comprehensively described by the numerical simulations of Hazel \& Heil (2002). Not surprisingly, comparison of the available results suggests that the dynamics of the system are rather different at low and high aspect ratios. At intermediate aspect ratios, we are aware of only three previous studies: (i) a lubrication-theory-based approach valid only at very low finger-propagation speeds (Wong, Radke \& Morris $1995 a, b)$; (ii) an investigation of the specific case of buoyancy-driven 'free' rise of bubbles (Clanet, Héraud \& Searby 2004); and (iii) our recent experimental results covering the range $1 \leqslant \alpha \leqslant 15$ (de Lózar, Hazel \& Juel 2007).

In this paper we extend the study of Hazel \& Heil (2002) and complement the experimental results of de Lózar et al. (2007) by solving the three-dimensional freesurface Stokes equations numerically in order to investigate the steady propagation of air fingers in rectangular tubes of intermediate aspect ratio $(1 \leqslant \alpha \leqslant 8)$. The chosen range of aspect ratios is the relevant one for microfluidics devices, in which microchannels are usually manufactured with $1 \leqslant \alpha \leqslant 10$ (Whitesides \& Stroock 2001). In the interest of simplicity, we neglect the effects of fluid inertia, but we do include the effects of a transverse gravitational body force for direct comparison with the experimental results. The agreement between our simulations and experiments is within the $\pm 1 \%$ experimental error, demonstrating the accuracy of the simulations and the quality of the experimental data.

The simulations provide detailed information about the influence of gravity and aspect ratio on the system. Furthermore, we uncover the physical origin of the empirical scaling found by de Lózar et al. (2007) relating the wet fraction (the relative quantity of liquid that remains on the walls after the propagation of the air finger) to a modified capillary number that depends on the aspect ratio. We show that, although not exact, this relation is valid to within experimental error over a wide range of parameter values. The present study also represents a significant step towards the direct numerical study of the Saffman-Taylor instability; and our simulations indicate that the Saffman-Taylor, quasi-two-dimensional, approximation is valid for $\alpha \geqslant 8$.

An outline of the paper is as follows. In $\S 2$, we describe the model problem and the numerical method, and present a validation of the numerical results and a discussion of the limitations of the simulation. In $\S 3$, we consider the effects of the aspect ratio $\alpha$ on the system in the absence of gravitational body forces, paying particular attention to the cross-sections of the air finger, the wet fraction, the pressure drop across the finger tip, the relative finger width and the topology of flow fields. In $\S 4$, we examine 


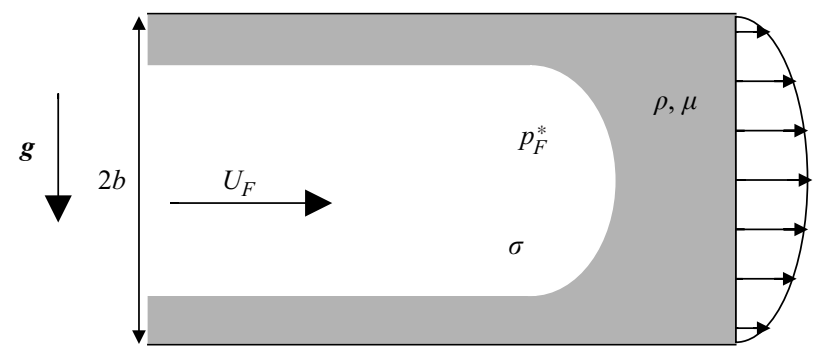

FiguRE 1. Sketch of the problem: an air finger propagates at a constant speed $U_{F}$ into a tube of rectangular cross-section with height $2 b$ and width $2 w$. The tube is initially filled by a wetting Newtonian fluid of viscosity $\mu$ and density $\rho$ and the surface tension at the air-liquid interface is $\sigma$. A gravitational body force of magnitude $g$ acts normally to the axis of the tube.

the effects of gravity on the system and in $\S 5$, we revisit the empirical scaling of de Lózar et al. (2007). Finally, we draw our conclusions in $\S 6$.

\section{The model}

A finger of air, assumed to be of negligible viscosity and density, propagates at a constant speed $U_{F}$ under an internal pressure $p_{F}^{*}$ into a tube of uniform rectangular cross-section with aspect ratio $\alpha=w / b$, where $2 b$ is the depth of the cross-section and $2 w$ is the width. Throughout this paper we use an asterisk to distinguish dimensional fluid pressures, fluid velocities, coordinates and curvatures from their dimensionless equivalents. The wetting fluid originally contained in the tube is assumed to be an incompressible Newtonian fluid of dynamic viscosity $\mu$ and density $\rho$ and the liquidair interfacial tension is given by $\sigma$. The entire system is subject to a gravitational body force of magnitude $g$ directed normally to both the direction of finger propagation and the wider plane of the tube's cross-section exactly as in de Lózar et al. (2007)'s experimental setup, see figure 1. For simplicity, we neglect inertial effects in the fluid.

\subsection{Governing equations}

The problem is formulated in dimensionless Cartesian coordinates $\boldsymbol{x}=\left(x_{1}, x_{2}, x_{3}\right)=$ $\boldsymbol{x}^{*} / b$ and the finger propagates in the negative $x_{2}$-direction. The outer walls of the tube are located at $x_{1}= \pm \alpha$ and $x_{3}= \pm 1$.

We non-dimensionalize the fluid velocity using the finger velocity $\boldsymbol{u}=\boldsymbol{u}^{*} / U_{F}$ and the fluid pressure using the viscous scale $p=p^{*} /\left(\mu U_{F} / b\right)$. In an inertial frame moving with the constant velocity of the finger the motion of the fluid is governed by the steady Stokes equations:

$$
-\frac{\partial p}{\partial x_{i}}+\frac{\partial}{\partial x_{j}}\left(\frac{\partial u_{i}}{\partial x_{j}}+\frac{\partial u_{j}}{\partial x_{i}}\right)+\frac{B o}{C a} k_{i}=0,
$$

and the equation of continuity

$$
\frac{\partial u_{j}}{\partial x_{j}}=0
$$

The Einstein summation convention is used and the indices take the values $i=1,2,3$. The vector $\boldsymbol{k}=(0,0,-1)$ indicates the direction of the gravitational body force. Two dimensionless parameters govern the behaviour of the system: the capillary number, $C a=\mu U_{F} / \sigma$ (the ratio of viscous to surface-tension forces) and the Bond number $B o=\rho g b^{2} / \sigma$ (the ratio of buoyancy to surface-tension forces). Hence, the 


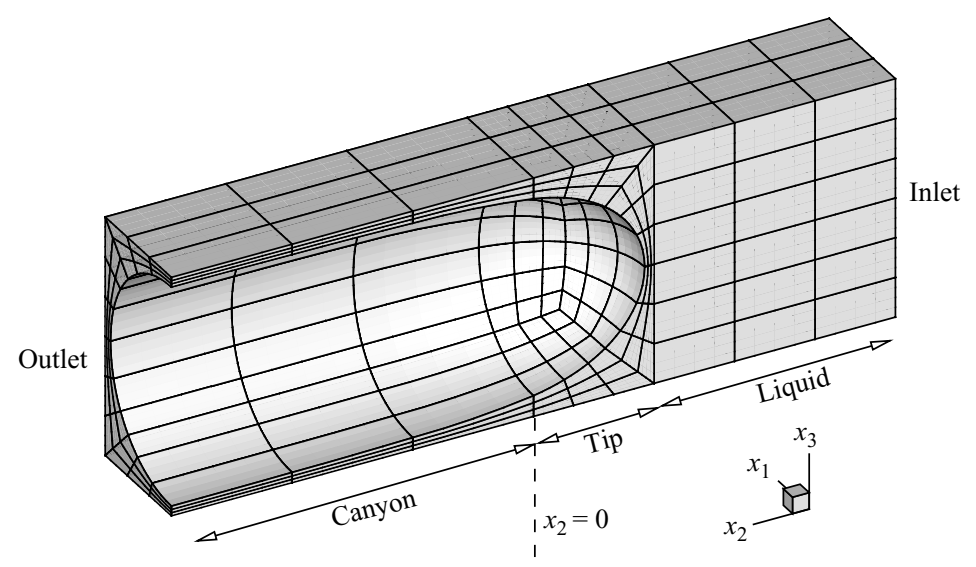

FIgURE 2. Illustrative (coarse) finite-element mesh. The mesh is divided into three subdomains: the liquid, tip and canyon regions. In the liquid subdomain, the mesh remains fixed. In the tip and canyon domains, the volume mesh deforms in response to changes in the interfacial position. The symmetry plane is $x_{1}=0$.

factor $\mathrm{Bo} / \mathrm{Ca}$ appearing in equation (2.1) represents the ratio of buoyancy to viscous forces, sometimes called a Galileo or Archimedes number.

\subsection{Boundary conditions}

On the free surface we impose a non-penetration condition

$$
u_{i} n_{i}=0,
$$

and a dynamic boundary condition for the jump in stress across the interface

$$
\left(\frac{\partial u_{i}}{\partial x_{j}}+\frac{\partial u_{j}}{\partial x_{i}}\right) n_{j}+\frac{1}{C a} \kappa n_{i}=\left(p-p_{F}\right) n_{i},
$$

where $\boldsymbol{n}$ is the unit vector normal to the free surface (directed out of the fluid) and $\kappa=\kappa^{*} b$ is the dimensionless mean curvature of the surface. On the tube walls we impose the non-slip boundary condition and in the moving frame of reference the wall velocity is

$$
\boldsymbol{u}=(0,1,0) .
$$

We must truncate our computational domain and require boundary conditions at the domain ends. Far behind the tip (at the outlet in figure 2), the variations in the axial direction are gentle enough that a long-wavelength approximation is valid. The usual assumption (see Reinelt $\&$ Saffmann 1985) is to neglect all axial variations at the end of the domain. Using this condition in equation (2.1) yields a Laplace equation for the axial velocity. Instead of this we impose a weaker condition: we assume that only the axial variations of the pressure and axial velocity are zero: $\partial p / \partial x_{2}=\partial u_{2} / \partial x_{2}=0$. This assumption permits axial variations in the transverse velocity components at the end of the computational domain and, although not rational in an asymptotic sense, was found to provide accurate results in smaller domains than those required when applying the 'full' long-wavelength approximation. Using this weaker condition in equation (2.1), we obtain

$$
\frac{\partial}{\partial x_{\beta}}\left(\frac{\partial u_{2}}{\partial x_{\beta}}+\frac{\partial u_{\beta}}{\partial x_{2}}\right)=0
$$


with $\beta=1,3$. The axial component of the momentum equation (2.1) is replaced by equation (2.6) at the outlet boundary subject to the boundary conditions of noslip at the tube wall and the approximate dynamic boundary condition on the free surface, obtained by taking the dot product of equation (2.4) with the unit vector in the $x_{2}$-direction and assuming that the vector normal to the free surface, $\boldsymbol{n}$, has no component in the $x_{2}$-direction. The approximate dynamic boundary condition is $\partial u_{2} / \partial n+\partial u_{n} / \partial x_{2}=0$, where $\boldsymbol{n}$ is now taken to be perpendicular to the free boundary in the plane of the boundary and $u_{n}$ is the velocity component directed along the vector $\boldsymbol{n}$. In the transverse directions, tractions consistent with the prescribed axial velocity and hydrostatic pressure gradient are applied.

Far ahead of the tip (at the inlet in figure 2), we enforce parallel flow $\left(u_{1}=u_{3}=0\right.$ ) and the hydrostatic pressure gradient $p=-x_{3} \mathrm{Bo} / \mathrm{Ca}$ is applied as a traction boundary condition in the axial direction. The axial velocity at the inlet, $u_{2}\left(x_{1}, x_{3}\right)$, is determined as part of the solution and we find that the axial velocity is approximately independent of the axial coordinate (centreline value varies by less than $1 \%$ ) by a distance $\alpha$ ahead of the finger tip. By imposing the pressure far ahead of the finger tip, the pressure within the air finger is no longer independent and $p_{F}$ must be treated as an unknown. The required additional equation is obtained by fixing the tip of the finger to remove the translational invariance of the problem.

\subsection{Numerical method}

The governing equations are solved numerically using a finite-element method via the object-oriented multi-physics library oomph-lib (Heil \& Hazel 2006). The fluid domain is divided into a number of volumetric elements, see figure 2. The symmetry of the problem allows us to restrict attention to half the fluid domain and symmetry boundary conditions are applied at $x_{1}=0$.

The deformation of the volume mesh in response to changes in the free surface is treated by the method of spines (Kistler \& Scriven 1983) and is essentially the same as that described by Hazel \& Heil (2002). In brief, the mesh is divided into three subdomains: the canyon, tip and liquid subdomains, see figure 2 . In the liquid domain, there is no free surface and so the mesh is fixed. In the canyon and tip regions, every node in the volume mesh is described by its fractional distance along a spine - a vector with a pre-determined direction, but unknown length $h$. In the tip region the spines are all directed towards the point $(0,0,0)$ and emanate from the tube walls or from the plane dividing the liquid and tip subdomains. In the canyon region, the spines lie in planes perpendicular to the axis of the tube and are directed from the tube walls to the centre of the tube. The spine lengths parameterize the position of the free surface and are determined during the solution procedure. As the free surface deforms, the nodal positions are updated in response to the changes in the spine lengths. A notable advantage of the scheme is that only a small number of nearby volume elements are remeshed in response to local changes in the freesurface position. We note in passing that the translational invariance of the problem is actually enforced by fixing the length of the spine directed along the axis of the tube. The end of this spine corresponds to the finger tip in the absence of gravity.

The selection of the axial extent of the mesh is not obvious because there are generally two different geometric length scales in the system: the height, 2, and width, $2 \alpha$, of the tube. In addition, the thickness of the fluid film deposited behind the finger tip sets another length scale. We note that the simulations are unable to resolve extremely thin films, which limits the range of capillary numbers that can be investigated, see $\S 2.5$. However, for high aspect ratios the system behaves like a 


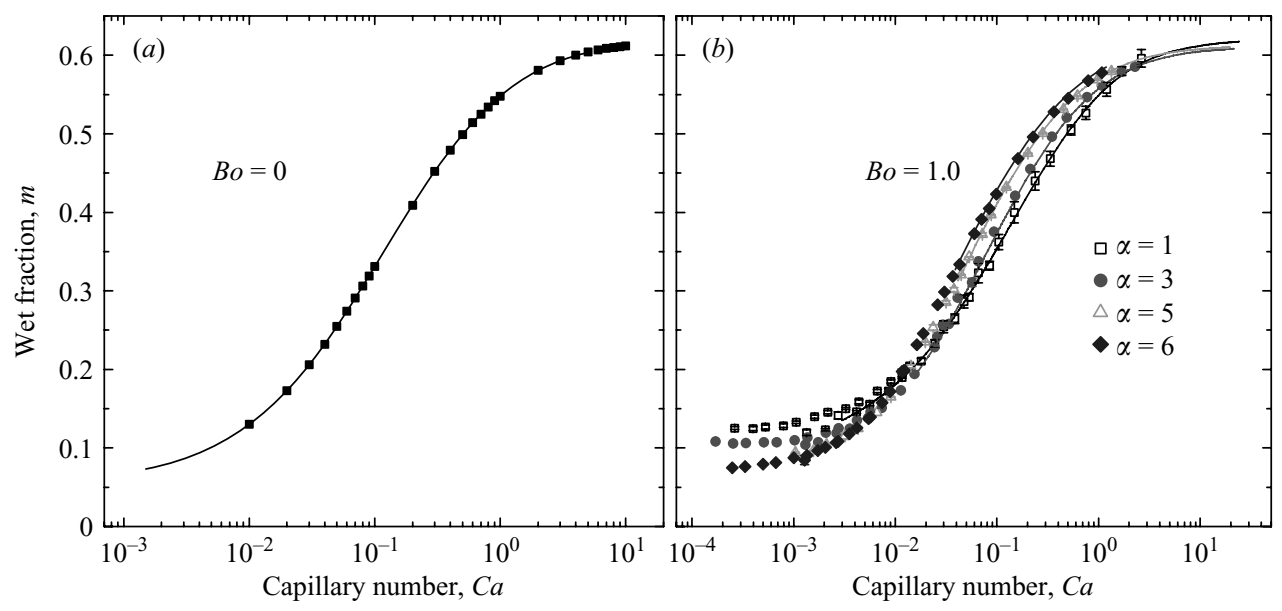

FIGURE 3. Wet fraction as a function of the capillary number: $(a)$ square tube at $B o=0$, the points represent the numerical data of Hazel \& Heil (2002) and are identical to our results to the three decimal places given in table 3 of their paper; $(b)$ rectangular tubes of aspect ratios $\alpha=1,3,5,6$ at $B o=1.0$, the experimental data of de Lózar et al. (2007) are shown as points and agree with our simulations to within the experimental error of $\pm 1 \%$. The development of thin films behind the finger tip sets the lower limit for $C a$ in our simulations, see $\S 2.5$, and the range of capillary numbers that can be accurately simulated decreases with increasing aspect ratio: $C a \geqslant 0.003(\alpha=1), C a \geqslant 0.016(\alpha=3), C a \geqslant 0.04(\alpha=5,6)$.

Saffman-Taylor system and $\alpha$ is the only important length scale (Reinelt 1987). For low aspect ratios $\alpha$ is equal to or longer than the typical length scale in the system. Therefore, we adjust the mesh length based on the tube width. Based on numerical experiments, the lengths of the liquid, tip and canyon submeshes were chosen to be $2 \alpha, \alpha$ and $3 \alpha$ respectively.

The fluid variables are discretized by using isoparametric $Q_{2} P_{-1}$ elements (Gresho \& Sani 2000), in which the quadratic fluid velocities are continuous, but the linear fluid pressures may be discontinuous between neighbouring elements. The spine lengths are discretized by isoparametric two-dimensional quadratic elements. The discrete system of equations is constructed by introducing the dynamic boundary condition (2.4) into the weak form of the momentum equations (2.1) as explained by Ruschak (1980); and the system is completed by the weak forms of the continuity equation (2.2), non-penetration boundary condition (2.3) and outlet boundary condition (2.6). The complete nonlinear system of equations is solved by Newton-Raphson iteration and the resulting linear system is assembled and solved by the HSLMA42 frontal solver (Duff \& Scott 1996). In our standard resolution we deal with 50000 degrees of freedom. An initial guess was generated by following the same procedure as Hazel \& Heil (2002) and once a converged solution had been found other solutions were obtained by slowly varying $\alpha, B o$ and $C a$.

\subsection{Validation of the code}

In figure $3(a)$ we compare our results with those of Hazel \& Heil (2002) in a square tube at $B o=0$. The wet fraction $m$ is the volume of fluid remaining in the tube after passage of the air finger divided by the volume of the tube. In our numerical simulations, the cross-sectional area of the fluid far behind the finger tip is given by the axial flow rate, $Q$, because $u_{2} \rightarrow 1$ as $x_{2} \rightarrow \infty$ where the fluid is at rest in the lab frame. The wet fraction is, therefore, calculated by the flow rate divided by 
the cross-sectional area of the tube, $m=Q /(4 \alpha)$. Our calculated wet fractions are indistinguishable from Hazel \& Heil's calculations to the three-digit precision given in table 3 of their paper. This comparison is important because although the method used for their simulations is very similar, the code is completely different. We also compare our results with the experiments of de Lózar et al. (2007), see figure 3(b). In the experimental setup, the system was driven by extracting fluid from the tube at a prescribed flow rate $Q_{F}$ and the wet fraction was taken to be $m=1-Q_{F} /\left(A U_{F}\right)$, where $A$ was the cross-sectional area of the tube and $U_{F}$ the measured velocity of the finger. All calculated wet fractions agree perfectly with the experimental results to within the experimental error of $\pm 1 \%$. We have also found good agreement with the results of Clanet et al. (2004) for the case of 'free' rise of bubbles in rectangular tubes whose axes are parallel to the direction of gravity, see the Appendix.

\subsection{The limit of the simulations: contact lines}

Far behind the finger tip, the fluid is at rest in the lab frame and the system is independent of the axial coordinate. Hence, the interface has a non-zero curvature in the transverse plane only and the curvature is proportional to the pressure difference across the interface. In the absence of gravity, the curvature must be a constant and the only possible interface shapes are arcs of circles. If the radius of curvature of the interface is greater than one, then the interface must intersect the tube walls because the fluid is wetting, i.e. the contact angle is zero. The final equilibrium configuration will, therefore, consist of fluid lobes connected by infinitely thin films. The inclusion of additional physical effects, such as disjoining pressures, would be required to model the eventual rupture of these films, and is outside the scope of the present study. The evolution towards the equilibrium configuration is driven by transverse draining flows that persist over long axial distances, whereas the axial velocity approaches its undisturbed value a relatively short distance behind the tip. The full details of the evolution dynamics were given by Wong et al. $(1995 a, b)$ in the limit of vanishing capillary number.

In figure 4 we show a cross-section of the flow domain at a distance $3.92 \alpha$ behind the tip for $C a=0.076, B o=0$ and aspect ratio $\alpha=3$. The axial velocity is approximately uniform and identical to the wall velocity $\left(\left|u_{2}-1\right|<0.001\right.$, relative error less than $0.1 \%)$. Nonetheless there are considerable pressure variations around the interface, driving a transverse flow from the fluid films above and below the finger to the edges of the tube, which causes a decrease in the finger width and concomitant thinning of the films with increasing axial distance from the tip.

The accurate numerical resolution of the evolution to equilibrium far behind the finger tip is precluded by the extreme thinning of the films, which leads to local under-resolution in our finite element method. At a fixed distance behind the finger tip the film thickness decreases with capillary number and the development of the thin films at the end of our computational domain sets the lower limit of capillary number in our simulations. The axial extent of the domain was chosen to be short enough that the films were sufficiently thick to be well-resolved, yet long enough so that the approximation of negligible axial variations in axial velocity and pressure was valid. A number of simulations in longer domains, in which the length of the canyon subdomain was doubled to $6 \alpha$, were performed and the bulk properties were found to be identical to those in our standard domain (differences of the order of $10^{-5}$ ).

Increasing the aspect ratio leads to a decrease in finger pressure at a fixed capillary number (Hazel \& Heil 2002), see also figure 6(b), associated with thinner fluid films. Hence, the range of capillary numbers that can be simulated decreases with increasing 


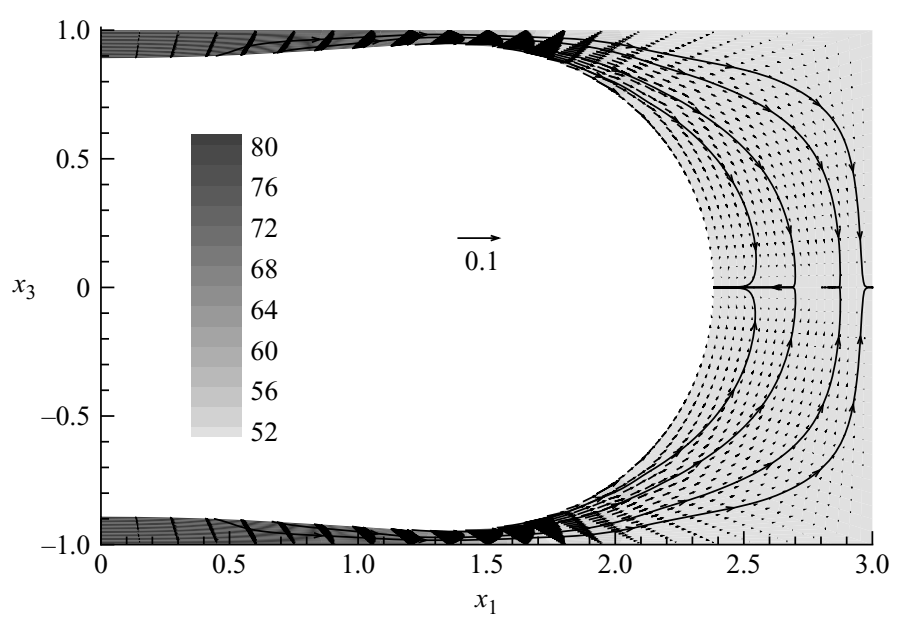

FIGURE 4. Cross-section of the flow domain at a distance $3.92 \alpha$ behind the tip for $C a=0.076$, $B o=0$ and aspect ratio $\alpha=3$. The grey scale represents the pressure on the surface-tension scale, the arrows show the transverse velocities of the liquid and the lines are streamlines. Two constant-pressure regions separated by a relatively thin liquid film are seen. The pressure drop between both regions drives a transverse flow that thins the intermediate region. Far from the tip the film becomes very thin, which eventually leads to a local under-resolution of our method.

\begin{tabular}{cccccccccc}
\hline & $\alpha$ & 1 & 2 & 3 & 4 & 5 & 6 & 7 & 8 \\
$B o$ & & & & & & & & & \\
0 & 0.002 & 0.003 & 0.003 & 0.005 & 0.010 & 0.015 & 0.015 & 0.015 \\
1.0 & 0.003 & - & 0.016 & - & 0.040 & 0.040 & 0.040 & - \\
2.5 & 0.005 & - & 0.022 & - & 0.15 & - & 0.15 & -
\end{tabular}

TABLE 1. The minimum simulated capillary number as a function of aspect ratio and Bond number. The maximum simulated capillary number was usually $C a=5$, apart from the validation runs presented in figure 3 when the maximum was $C a=20$.

aspect ratio. The maximum value of the aspect ratio in the simulations was $\alpha=8$. For larger values of $\alpha$, it was not possible to obtain results at sufficiently low values of $\mathrm{Ca}$ to explore the parameter regime associated with the single-finger Saffman-Taylor regime. Table 1 shows the minimum values of $C a$ in our simulations for different values of $\alpha$ and $B o$. For $\alpha=7$, we confirmed that the relative error in the bulk properties remained less than $0.1 \%$ on comparison between our standard resolution of 50000 degrees of freedom and a refined mesh of 60000 degrees of freedom.

\section{The influence of aspect ratio}

In this section, we shall discuss the influence of the aspect ratio on the system in the absence of any gravitational effects $(B o=0)$. Initially, we discuss changes in the cross-section of the air finger behind the finger tip, $\S 3.1$. We next turn our attention to bulk properties of the flow, $\S 3.2$, presenting the wet fraction, the pressure drop across the finger tip and the relative finger width as functions of aspect ratio and capillary number. Finally, in $\S 3.3$ we discuss the flow fields and the generic topological changes that occur with variations in the capillary number and aspect ratio. 

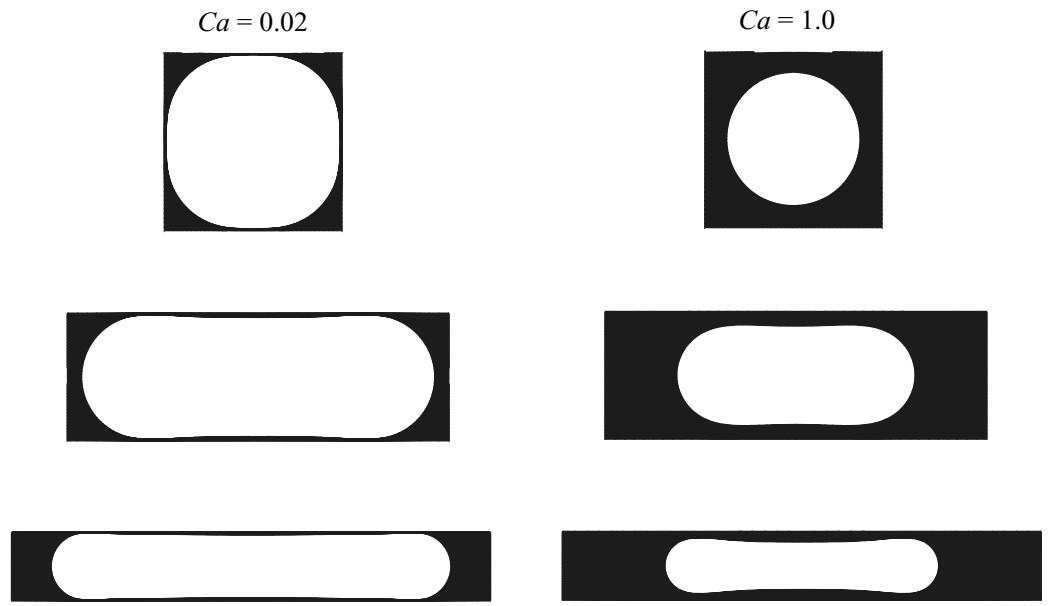

$\alpha=7$

Figure 5. Cross-sections of the flow domain for two capillary numbers $(C a=0.02,1.0)$, three aspect ratios $(\alpha=1,3,7)$ and $B o=0$, at a distance of $3.92 \alpha$ behind the tip.

\subsection{The cross-section of the air finger}

In figure 5 we show cross-sections of the flow domain at a distance of $3.92 \alpha$ behind the tip for two capillary numbers $(C a=0.02,1.0)$ and three aspect ratios $(\alpha=1,3,7)$. The axial velocity of the fluid is almost uniform and is within $0.1 \%$ of the wall velocity for the parameter values shown.

At $C a=0.02$ and $\alpha=1$, the cross-sectional flow domain consists of four corner regions of constant curvature, connected by thin films. As discussed in $\S 2.5$, the difference in curvature between the corner regions and the thin films generates a pressure drop that drives transverse draining flows from the films into the corner regions. The volume of fluid transported, however, is so small that it does not measurably deform the interface, and thus the air finger is in quasi-equilibrium. In fact, a fluid domain consisting of four corner regions of constant curvature is an equilibrium configuration at $C a=0$ for any value of $\alpha$ (Wong et al. 1995a). Experimental evidence (Moore et al. 2002) suggests that these configurations may be unstable for very high aspect ratios $\alpha \gtrsim 250$, however.

At $C a=0.02$ and $\alpha=3$ and 7, the quasi-equilibrium configuration is altered compared to $\alpha=1$. The interface consists of two end regions of constant curvature along the short semi-axes of the tube, connected by two thin liquid films along the longer semi-axes. The thin films ensure that the draining flows are weak; the maximum magnitude of the transverse velocity, $\sqrt{u_{1}^{2}+u_{3}^{2}}$, is approximately $0.05(0.048$ for $\alpha=3$ and 0.054 for $\alpha=7$ ) and the minimum film thickness is approximately 0.01 , giving an estimate of the strength of the draining flow as 0.0005 , much smaller than the order-one axial flow. Hence, the variation of the finger width in the axial direction is negligible (in both cases, the values of dimensionless finger width $\lambda$, defined in $\S 3.2$, measured at cross-sections located at $3.92 \alpha$ and $2.92 \alpha$ behind the finger tip differ by less than $\left.8 \times 10^{-4}\right)$. McLean \& Saffman (1981) and Reinelt (1987) used this crosssectional configuration to derive the boundary conditions for their two-dimensional simulations of the Saffman-Taylor instability.

As $\mathrm{Ca}$ increases, the fluid films thicken and in near-square tubes the ultimate finger shape becomes axisymmetric at sufficiently high $\mathrm{Ca}$ (Ratulowski \& Chang 1989; Kolb \& Cerro 1991). Hazel \& Heil (2002) showed that these axisymmetric 

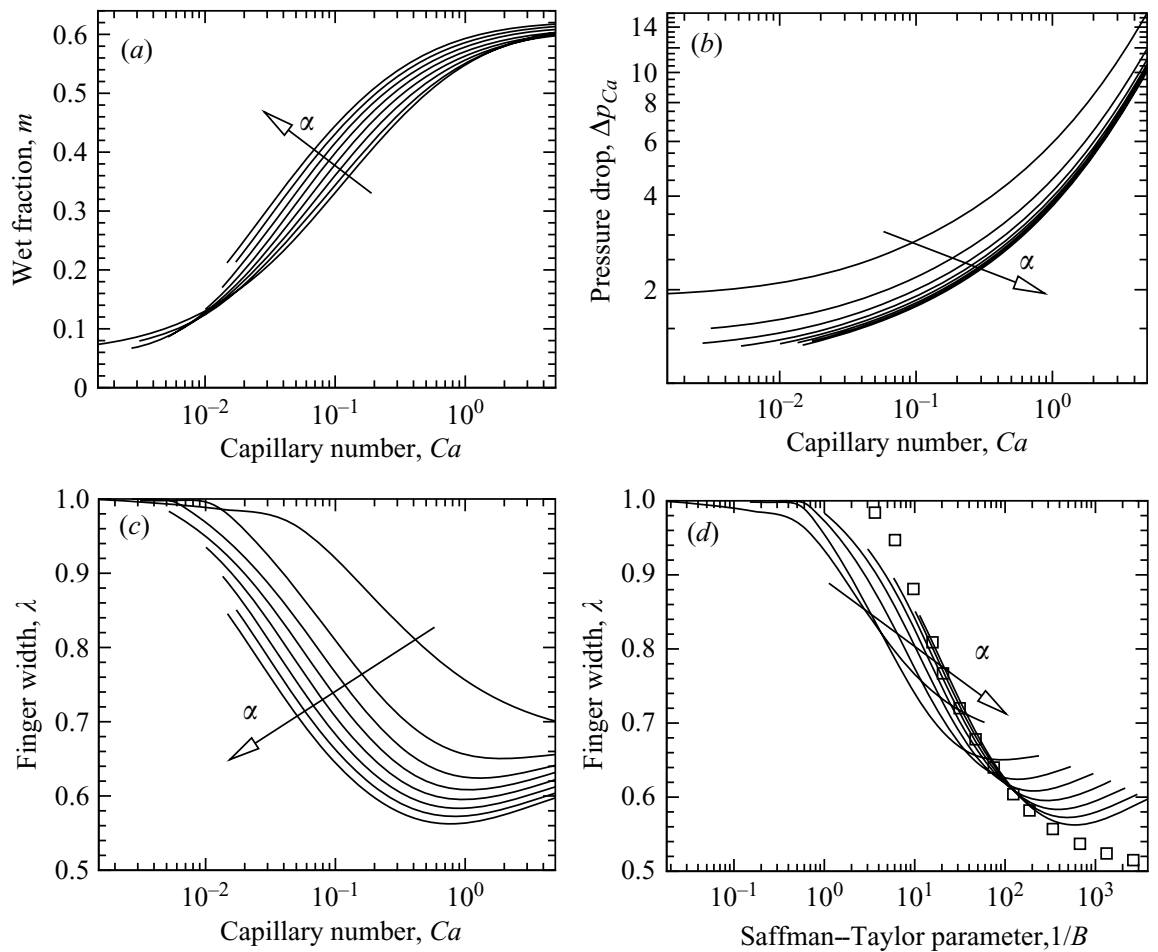

Figure 6. (a) The wet fraction, $(b)$ pressure drop, and (c) relative finger width plotted against the capillary number for tubes of aspect ratios $\alpha=1,2 \ldots, 8$ in the absence of gravity $(B o=0)$. (d) The relative finger width is also plotted as a function of the Saffman-Taylor parameter $1 / B=12 \alpha^{2} C a$ together with the results of the two-dimensional simulations of McLean \& Saffman (1981) (squares). The data are taken from table 1 in their paper, but we have omitted the point corresponding to $\kappa=3.09$ because it appears to be in error on comparison with their figure 4. Note that in $(b)$ and $(d)$ the pressure drops and finger widths (for $1 / B<200$ ) are almost indistinguishable for $\alpha=7$ and $\alpha=8$. The finger widths for these values of $\alpha$ are in excellent agreement with the results of McLean \& Saffman (1981) for $1 / B \leqslant 200$, suggesting that tubes with $\alpha \geqslant 8$ support Saffman-Taylor finger propagation.

configurations were possible for $\alpha \lesssim 2.04$, but for $\alpha \gtrsim 2.04$ the axisymmetric configuration never occurs. Instead, the equilibrium finger shape consists of end regions of constant curvature connected by thick films along the longer semi-axes of the tube, as shown for $C a=1.0$ and $\alpha=3,7$. The thick films enhance the effect of the draining flows, resulting in a stronger axial variation of the finger width within our finite flow domain. ( $\lambda$ measured at cross-sections $3.92 \alpha$ and $2.92 \alpha$ far from the tip differ by $8 \times 10^{-3}$ for $\alpha=3$ and by $3 \times 10^{-3}$ for $\alpha=7$.) The finger is still expected to relax into a quasi-equilibrium configuration similar to that shown at $C a=0.02$, but only at very long distances from the tip (not seen in our simulations).

\subsection{Bulk properties}

In figure 6 we present results for tubes of aspect ratios $\alpha=1,2 \ldots, 8$ in the absence of gravity $(B o=0)$. The wet fraction $m$, defined in $\S 2.4$, increases with aspect ratio at high $C a$, while at low $C a$ the opposite tendency is observed, see figure 6(a). The behaviour of $m$ at low $C a$ could be determined only for $\alpha \leqslant 4$ owing to the limitations imposed by the development of the thin films in high-aspect-ratio tubes, but has been observed experimentally at higher aspect ratios by de Lózar et al. (2007). These 
opposite variations of $m$ with aspect ratio are associated with different interface shapes far behind the finger tip, see figure 5 and $\S 5.1$. In the limit of low $C a$, and indeed for $C a=0$ (Wong et al. 1995a), the final interface shape consists of four corner regions, whose constant curvature is not imposed by the geometry of the tube. Thus, the wetted area does not alter significantly with increasing $\alpha$, resulting in decreasing values of $m$. For higher $C a$, the fluid domain far behind the finger tip consists of two 'side' regions, whose constant curvature is set by the shorter semi-axis of the tube's cross-section. The width of these regions grows more rapidly with $\alpha$ than the width of the finger, resulting in a net increase in $m$.

In figure $6(b)$, we plot the pressure drop across the tip of the finger on the capillary scale $\left(\Delta p_{C a}=\Delta p^{*} /(\sigma / b)\right)$ as a function of the capillary number. The pressure drop decreases with the aspect ratio, reflecting a reduction in tip curvature that is associated with widening tubes, but the separation between the curves is also reduced as $\alpha$ increases. Thus, the pressure drops for $\alpha=7$ and $\alpha=8$ are almost indistinguishable as the system approaches the Saffman-Taylor regime. At high enough $\alpha$ the curvature at the tip (which determines $\Delta p_{C a}$ ) is dominated by the curvature across the tube (of order $1 / b$ ), which depends only weakly on the aspect ratio. For very high $\alpha$ and asymptotically low $C a$, Park \& Homsy (1984) predicted a weak dependence of $\Delta p_{C a}$ on $\alpha$, of the form of $1 / \alpha^{2}$. Our simulations suggest that the $\alpha$ dependence of $\Delta p_{C a}$ weakens further as $C a$ increases.

In the literature, the typical parameter used to characterize air fingers in rectangular tubes of high aspect ratio is the relative finger width, $\lambda$, the width of the air finger divided by the width of the tube's cross-section. This quantity appears naturally in the two-dimensional model and is easy to measure experimentally. The definition of $\lambda$ in the three-dimensional system is not clear, however. Based on what would be observed in experiments we define the relative finger width to be the maximum $x_{1}$-coordinate of the finger divided by the aspect ratio. The measurement is taken in the last $x_{1} x_{3}$-cross-section of our computational domain, at a distance of $3.92 \alpha$ behind the tip. An example cross-section at $C a=0.076$ is shown in figure 4, for which the relative finger width is $\lambda=0.795$. At this distance from the tip, $\lambda$ is independent of the axial coordinate for low $C a$. At high $C a$, however, the finger width does not stabilize until much greater distances. This is because the relatively weak surface-tension forces require longer axial length scales to restore the interface to a quasi-equilibrium state after the distortion caused by the non-axisymmetric passage of fluid around the tip, as explained by Hazel \& Heil (2002).

Figure 6(c) shows the relative finger width plotted as a function of the capillary number $C a$. The finger width decreases with $C a$ as expected, but for sufficiently high $\mathrm{Ca}$, the width reaches a minimum and starts to increase, a consequence of measuring the width at a fixed axial distance behind the tip. At high values of the capillary number the finger's cross-section is no longer independent of the axial coordinate at our chosen distance. We expect that the finger width measured sufficiently far behind the tip does indeed decrease monotonically with increasing $\mathrm{Ca}$, as found by Tabeling, Zocchi \& Libchaber (1987) in experiments at high aspect ratios. Increasing the aspect ratio leads to reduced relative finger widths for all $\mathrm{Ca}$ because the curvature, and hence the surface-tension forces, decrease as $\alpha$ is raised.

In figure $6(d)$ we show the relative finger width plotted as a function of the Saffman-Taylor parameter $1 / B=12 \alpha^{2} C a$. This is the typical plot shown in the literature to characterize the Saffman-Taylor instability, see Homsy (1987). Again, the separation between the curves decreases as the aspect ratio increases and the curves corresponding to $\alpha=7$ and $\alpha=8$ are very close for $1 / B<200$. These findings 
are in agreement with the experimental results of de Lózar et al. (2007), who found that at fixed $1 / B$ the relative finger widths were identical to within experimental error over the range $8 \leqslant \alpha \leqslant 15$, suggesting that the system is Saffman-Taylor-like for $\alpha \gtrsim 8$. Moreover, we find quantitative agreement at $\alpha=8$ with the two-dimensional simulations of McLean \& Saffman (1981) for $10 \leqslant 1 / B \leqslant 200$, where the relative error is less than $1.5 \%$. The separation of the curves for $1 / B>200$ is a consequence of the dependence of $\lambda$ on axial distance, as explained above. To our knowledge, this is the first evidence of quantitative agreement between two- and three-dimensional models of these systems, and our results suggest that tubes with $\alpha \geqslant 8$ support Saffman-Taylor finger propagation.

The values of $C a$ and $1 / B$ for which $\lambda$ reaches its minimum in figures $6(c)$ and $6(d)$, respectively, give an indication of the parameter thresholds beyond which the interface does not reach a quasi-equilibrium configuration within our simulation domain. Whereas the threshold value of $C a$ decreases with $\alpha$, the corresponding value of $1 / B$ increases with $\alpha$. This explains why $\lambda$ has not been found to vary significantly with the distance from the finger tip in Saffman-Taylor experiments, which are performed at much higher aspect ratio than our simulations $(\alpha \gtrsim 20)$. However, in low-aspect-ratio tubes the relaxation of the interface must be taken into account when defining $\lambda$, which generally depends on the distance from the finger tip.

\subsection{Flow fields}

The flow field associated with the propagating air finger in rectangular tubes has important similarities with its analogue in cylindrical tubes. Taylor (1961) showed that, at sufficiently low flow rates, the velocity of the fluid near the centreline of a cylindrical tube is faster than the finger velocity, owing to the existence of a recirculating flow in the moving frame of reference. The centreline velocity changes sign for increasing flow rates (i.e. increasing $\mathrm{Ca}$ ), resulting in the disappearance of the recirculation region ahead of the finger. In the cylinder, the transition between these two flows involves two topological changes that were conjectured by Taylor (1961) and observed by Giavedoni \& Saita (1997). In near-square tubes, Hazel \& Heil (2002) identified an additional non-axisymmetric regime, resulting in four generic flow regimes. For larger aspect ratios, $\alpha \geqslant 3$, a total of six flow regimes are generic as illustrated in figure $7(a-f)$ for $\alpha=5$, where streamlines are plotted both on the horizontal plane of symmetry $\left(x_{3}=0\right)$ and on the free surface. At low capillary numbers, figure $7(a)$, the flow is characterized by three stagnation points (five in the whole tube) located on the free surface: one at the tip of the finger and one in each symmetry plane (horizontal, $x_{3}=0$ and vertical, $x_{1}=0$ ). This flow regime is similar to that observed in rectangular tubes of smaller aspect ratio (Hazel \& Heil 2002). The first topological change observed when increasing $\mathrm{Ca}$ is the splitting of the stagnation point in $x_{3}=0$, which leads to a new stagnation point migrating ahead of the finger, figure 7(b). When increasing $\mathrm{Ca}$ further, the original stagnation point in $x_{3}=0$ moves around the finger to merge with the tip stagnation point, figure $7(c)$. The stagnation point in $x_{3}=0$ ahead of the finger also migrates toward the centre of the tube. Once its reaches the centreline, the flow ahead of the stagnation point is reversed and the associated 'global' recirculation flows vanish, figure $7(d)$. A localized recirculation remains, however, between the tip and the axial stagnation point. This new regime, which is typical of higher-aspect-ratio tubes $(\alpha \geqslant 3)$, may be of practical interest, as the finger tip is effectively shielded by the localized recirculation flow. Further increases in $\mathrm{Ca}$ lead to the merging of the axial stagnation point with the tip and the associated disappearance of the localized recirculation flow, figure 7(e), followed 

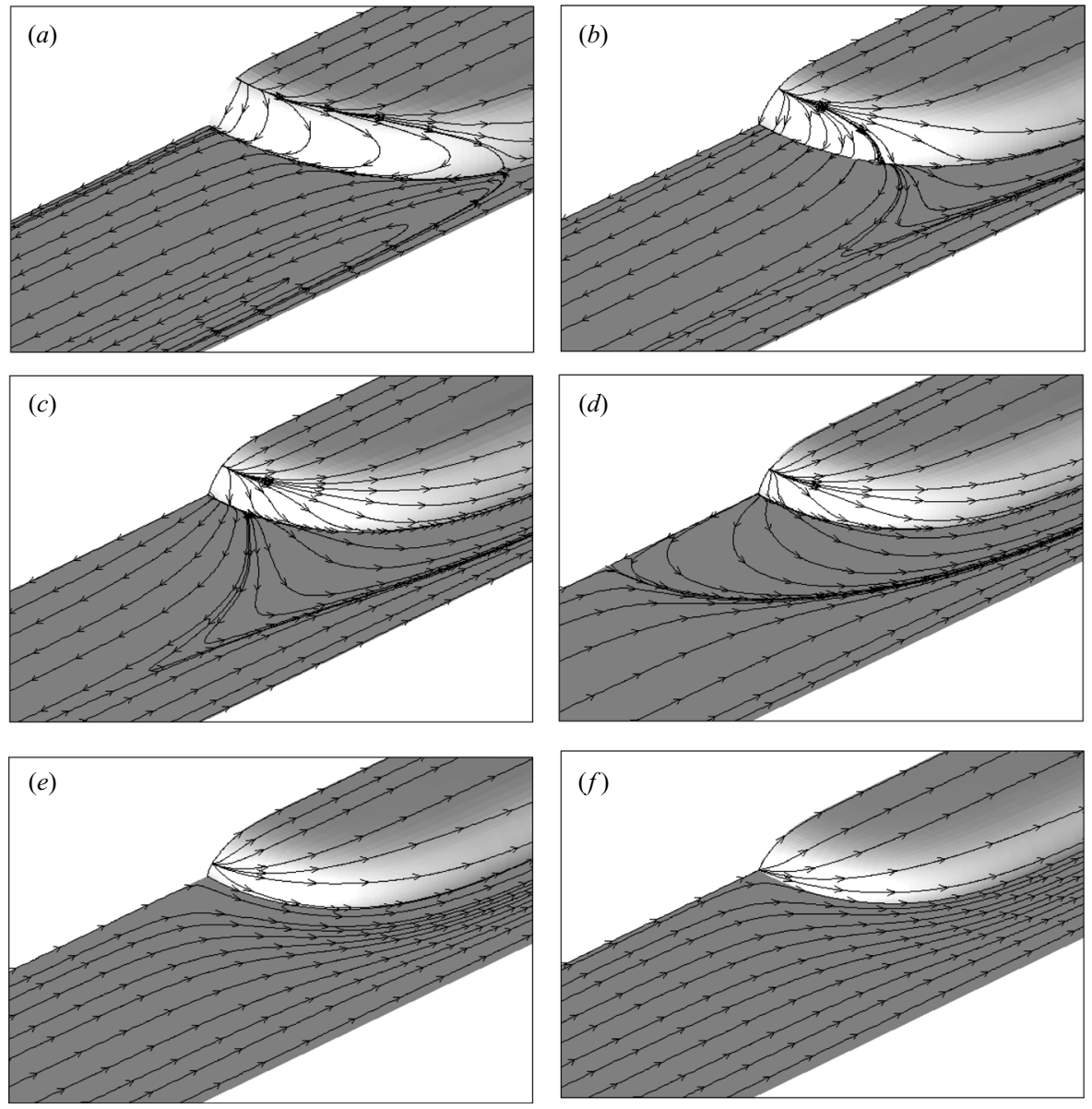

FiguRE 7. Streamlines in the moving frame of reference for a rectangular tube $\alpha=5$ and $B o=0$. (a) $C a=0.0111$, (b) $C a=0.0441$, (c) $C a=0.0816$, (d) $C a=0.122$, (e) $C a=0.292$, (f) $\mathrm{Ca}=0.792$. Streamlines are shown in the horizontal symmetry plane $x_{3}=0$ and on the free surface.

by the migration of the stagnation point in $x_{1}=0$ to merge with the tip stagnation point, figure $7(f)$. This high- $C a$ flow is topologically equivalent to that observed in the cylindrical and square tubes.

The transition between regimes $(c)$ and $(d)$, or the equivalent regimes at low aspect ratios, corresponds to the disappearance of the 'global' recirculation flow. Figure 8 shows the capillary number at which this transition occurs, $C a_{c}$, as a function of the aspect ratio. The error bars and symbols represent the range and average value of $C a$, respectively, based on the largest value of $C a$ simulated for which recirculation flows were observed, and the lowest value of $C a$ simulated, for which they could not be seen. Excluding the measurements in the square tube, $\alpha$ exhibits a power-law dependence on $C a_{c}$ of the form, $C a_{c}^{\beta} \alpha=A$, where $A=1.21 \pm 0.01$ and $\beta=0.66 \pm 0.01$ were obtained from a least-square linear fit of the logarithmic data. Note that a lubrication analysis of thin films implies that the film height scales with $C a^{2 / 3}$ (Landau \& Levich 1942), suggesting that the topological change in the flow indicated by $C a_{c}$ is directly associated with the flow resistance of the thin films present at the top and bottom of the tube. This explanation is consistent with the deviation observed at low $\alpha$, when 


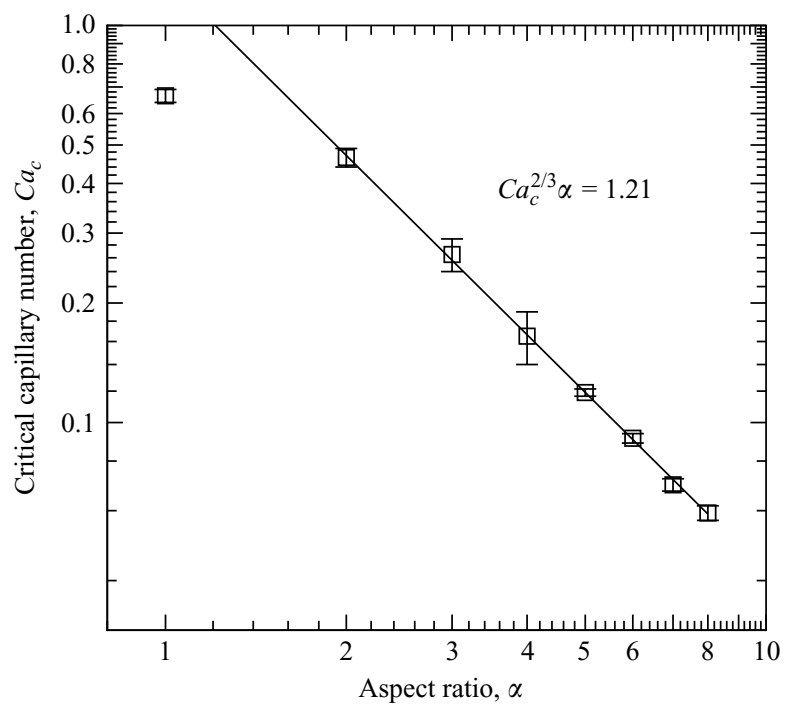

FIGURE 8. Capillary number at which the recirculation flows disappear as a function of the aspect ratio. The line is a linear fit that gives $C a_{c}^{2 / 3} \alpha=1.21$.

the change of topology in the square and near-square tubes occurs at large enough values of $C a_{c}$ that the films are not thin.

\section{The effect of gravity}

We focus on the influence of transverse gravity (along the $x_{3}$-direction) that would apply to experiments performed in a horizontal tube (de Lózar et al. 2007). The effect of gravity is most pronounced at low $\mathrm{Ca}$, where viscous forces are relatively weak. For a fixed $B o$, the buoyancy-force term in equation (2.1) decreases in magnitude with increasing $\mathrm{Ca}$ and so the influence of gravity on the system is negligible at high $\mathrm{Ca}$.

In figure 9 we show cross-sections of the flow domain at a distance of $3.92 \alpha$ behind the tip for two values of the Bond number $(B o=1.0,2.5)$ and three aspect ratios $(\alpha=1,3,7)$ at $C a=0.2$. In the presence of transverse gravity the hydrostatic pressure that increases linearly with distance from the upper wall of the tube must be balanced by the pressure drop across the free surface, in order for the pressure inside the finger to remain constant. Thus, the curvature of the free surface decreases with distance from the upper wall. For $\alpha=1$, as the Bond number is raised from $B o=1.0$ to 2.5, the finger becomes increasingly distorted from the circular configuration adopted when $B o=0$. In tubes of higher aspect ratios $(\alpha=3,7)$, it is the end regions, of constant curvature when $B o=0$, that deform to balance the hydrostatic pressure. Jensen et al. (1987) described the resulting fingers as 'banana shaped'.

Jensen et al. (1987) also demonstrated that for $B o>1$ static interface shapes were possible in which the air finger lifts off the bottom wall of the tube, because surfacetension forces cannot balance the hydrostatic pressure across the entire depth of the tube. As a result, in our simulations, the bottom film is much thicker than the top for $B o=2.5$, compared to the case when $B o=1.0$. In the bottom part of the tube, the effect of the hydrostatic pressure is to drive a flow toward the bottom film, thus opposing the draining flow due to curvature changes. As fluid is transported from the upper part of the flow domain toward the bottom, the finger lifts and widens 

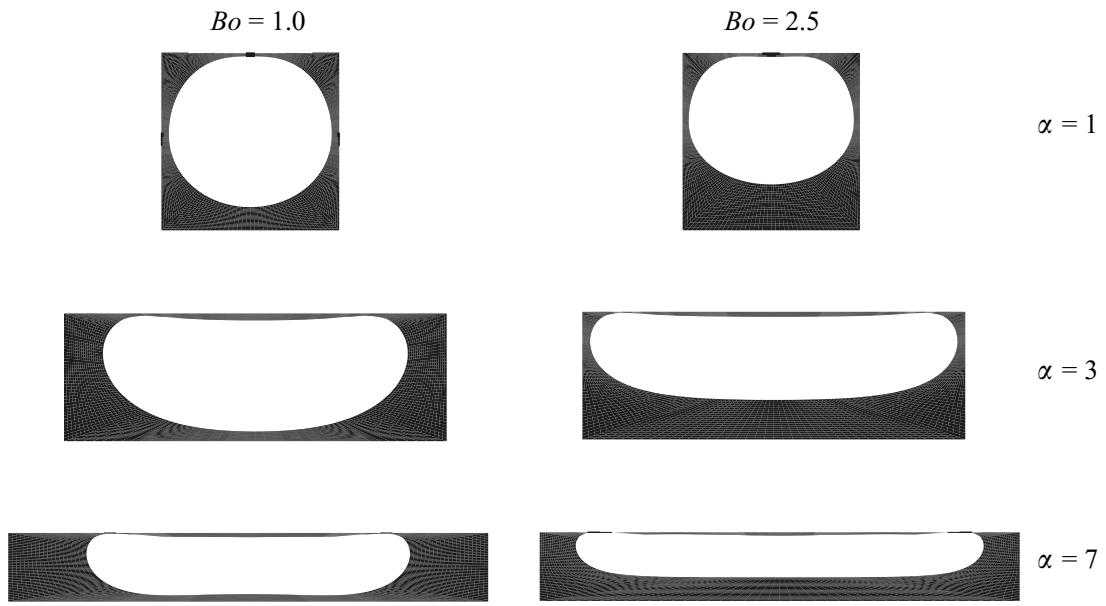

Figure 9. Cross-sections of the flow domain at a distance of $3.92 \alpha$ behind the tip and $C a=0.2$, for $B o=1.0$ and 2.5 , and three values of the aspect ratio $(\alpha=1,3,7)$. The finger lifts off the bottom wall for $B o=2.5$.

with distance from the tip until a quasi-equilibrium configuration is reached. The enhancement of the transverse flows by the hydrostatic pressure gradient means that the finger widths stabilize over shorter axial distances, but that the upper film is thinner than the equivalent case in the absence of gravity. Hence, the range of $\mathrm{Ca}$ that can be accurately simulated is reduced as $B o$ increases.

We note that for intermediate values of the capillary number (large enough that the interface is not influenced by the sidewalls of the tube, but small enough that the interface will eventually meet the upper wall) the ultimate equilibrium cross-sections will be the same as those of two-dimensional sessile droplets resting on a flat plate under the influence of gravity. In these cases, the axial evolution of the pendent film on a length scale of $\mathrm{Ca}^{-1}$, see Wong et al. (1995a), should be similar to the temporal evolution of two-dimensional droplets settling onto a solid surface on the surface-tension time scale, as described by Lister, Morrison \& Rallison (2006). Our computational domains were not sufficiently long to capture such dynamics, however.

The reduction in the curvature of the free surface in the presence of transverse gravity also means that the finger occupies a smaller proportion of the cross-section of the tube. Hence, at fixed $\mathrm{Ca}$, the wet fraction increases with $B o$, as shown in figure $10(a, b)$ for $\alpha=1$ and $\alpha=3$, respectively. The considerable increase in the wet fraction between $B o=1.0$ and $B o=2.5$ is again a consequence of the change in equilibrium shape for $B o>1$, above which the cross-sectional area of the finger decreases rapidly with increasing $B o$. The finger tip is defined as the point furthest ahead on the free surface and as the finger lifts with increasing $B o$, so does its tip, leading to an enhanced pressure drop at the tip, see figure 10(c). Finally, the increase in width of the finger with increasing $B o$ is shown in figure $10(d)$. The behaviour of the bulk properties is similar for all aspect ratios and for clarity we present results at a single aspect ratio $\alpha=3$. In figure $10(d)$ we also present experimental finger widths $(B O=1)$ measured at the same distance behind the finger tip as the end of our computational domain (unpublished data from the experiments of de Lózar et al. 2007). The agreement between the simulations and experimental data is excellent (within the $\pm 1 \%$ experimental error). 

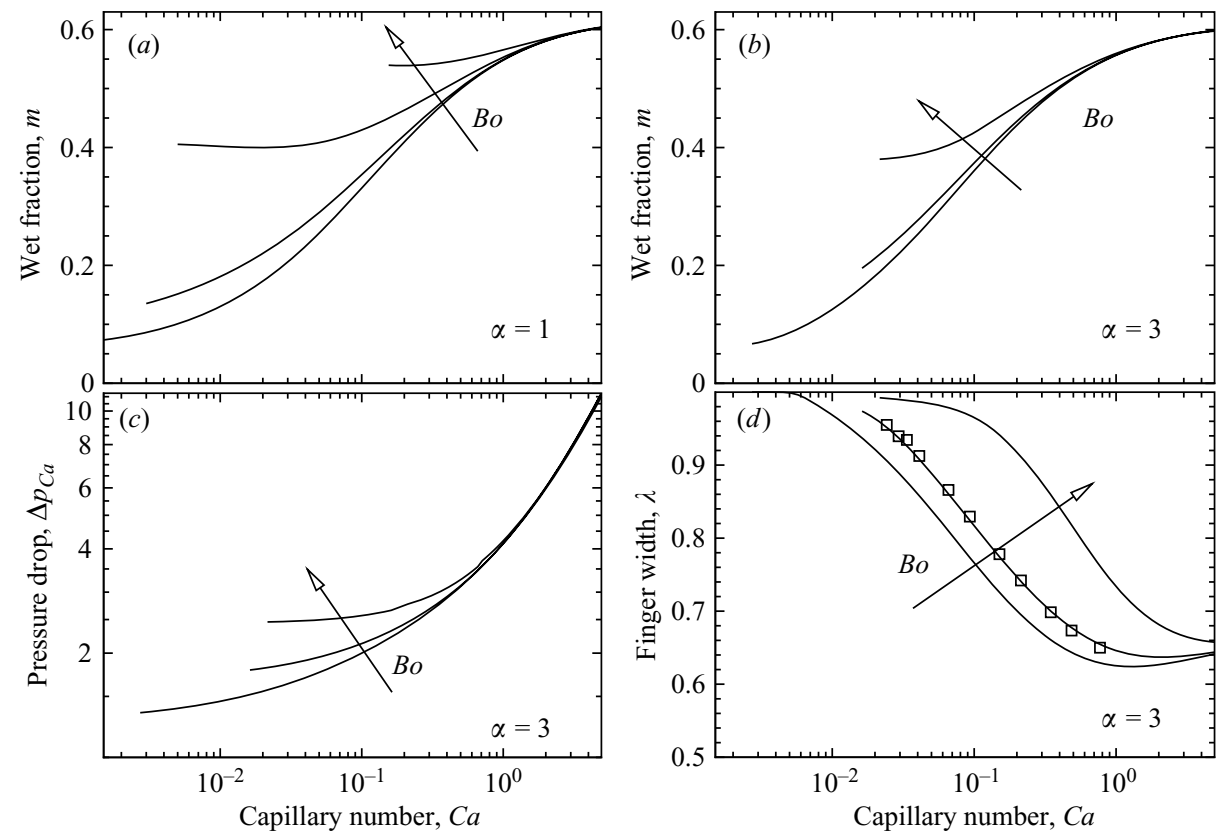

FIGURE 10. Wet fraction as a function of the capillary number for the aspect ratios $(a)$ $\alpha=1$ and $(b) \alpha=3$. For $\alpha=3$, we also show $(c)$ the pressure drop and $(d)$ relative finger width as a function of the capillary number. The symbols in $(d)$ correspond to experimental measurements of the finger width taken at the same distance behind the finger tip as the end of the computational domain (unpublished data from the experiments of de Lózar et al. 2007). The lines correspond to different values of the Bond number $(B o=0,1.0,2.5)$. In the wet fraction for the square tube $(a)$ the results for $B o=5$ are also presented. Note the considerable increases in the wet fraction and finger width between $B o=1.0$ and $B o=2.5$ compared to those between $B o=0$ and $B o=1.0$.

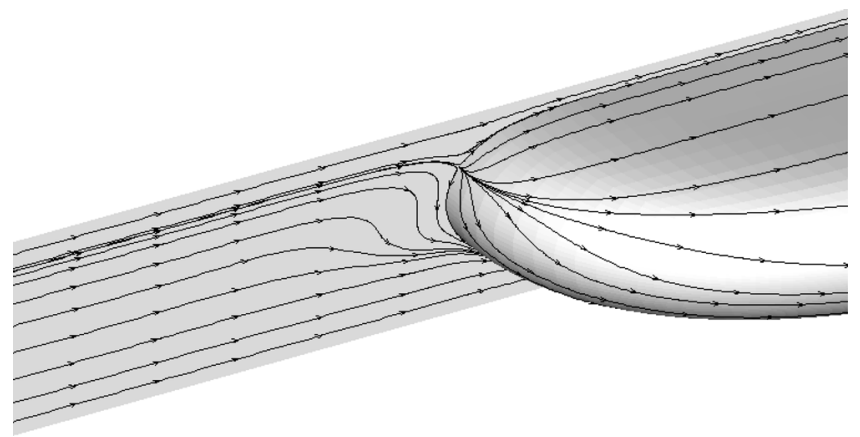

Figure 11. Stream lines in the moving frame of reference at $\alpha=5, C a=0.292$ and $B o=1.0$. Stream lines are plotted in the vertical symmetry plane, $x_{1}=0$, and on the bubble surface.

The presence of gravity breaks the reflection symmetry about $x_{3}=0$, and complicates the topology of the flow considerably. The flow field remains simple for large $C a$, however, where it is characterized by a single stagnation point, as shown in figure 11 for $C a=0.292$ and $B o=1.0$. This flow regime is topologically analogous to that seen in the absence of gravity shown in figure $7(f)$, but the stagnation point is located above the finger tip rather than exactly at the tip as for $B o=0$. Increasing $\mathrm{Ca}$ causes the stagnation point to migrate closer to the tip as the influence of gravity 

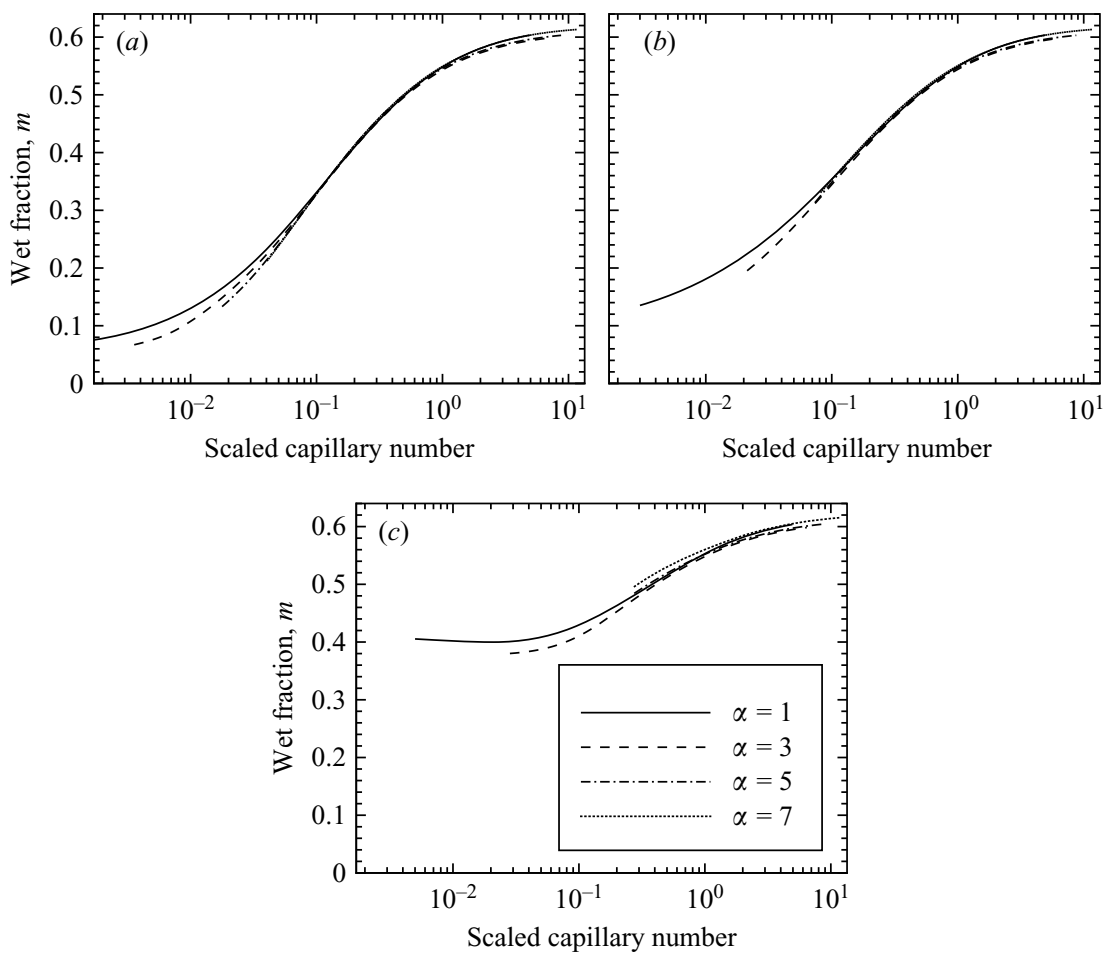

FIGURE 12. Wet fraction as a function of $\widehat{C a}=C a\left[1+a_{1}(\alpha-1)+a_{2}(\alpha-1)^{2}\right]$, the scaled capillary number, with experimentally determined parameter values (de Lózar et al. 2007). The lines correspond to aspect ratios $\alpha=1,3,5,7$. (a) $B o=0$, (b) $B o=1,(c) B o=2.5$.

decreases. Non-zero gravity also reduces the minimum value of the capillary number for which the flow regime defined by one stagnation point is observed. At $C a=0.292$, the flow field at $B o=1.0$ includes one stagnation point, figure 11 , whereas at $B o=0$, it has three, figure $7(e)$.

\section{The empirical scaling of de Lózar et al. (2007)}

One of the most surprising results of the experimental study of de Lózar et al. (2007) was the discovery that the wet fraction appeared to depend on a single modified capillary number $\widehat{C a}=C a\left[1+a_{1}(\alpha-1)+a_{2}(\alpha-1)^{2}\right]$ for all aspect ratios above $\widehat{C a}>\widehat{C a}_{t} \approx 0.035$. The scaling has obvious practical applications, but its origin is strictly empirical and it was determined from a set of experiments conducted at a single Bond number, $B o=1.0$.

In figure 12 the wet fraction obtained in tubes of $\alpha=1,3,5$ and 7 is plotted against the scaled capillary number, using the experimentally determined parameters $\left(a_{1}=0.12 \pm 0.02\right.$ and $\left.a_{2}=0.018 \pm 0.001\right)$ for three different values of the Bond number. The curves never collapse perfectly, but the wet fractions differ by less than 0.013 for $\widehat{C a}_{t}>0.05$ at $B o=0$. Remarkably the curves are almost indistinguishable over one order of magnitude in $\widehat{C a}(0.1 \leqslant \widehat{C a} \leqslant 1)$. Therefore, although not exact, the error in predictions made using the effective scaling is approximately half that of the typical experimental error $( \pm 0.01)$ given by de Lózar et al. (2007). Following the same least-squares procedure as de Lózar et al. (2007), but using the data from 


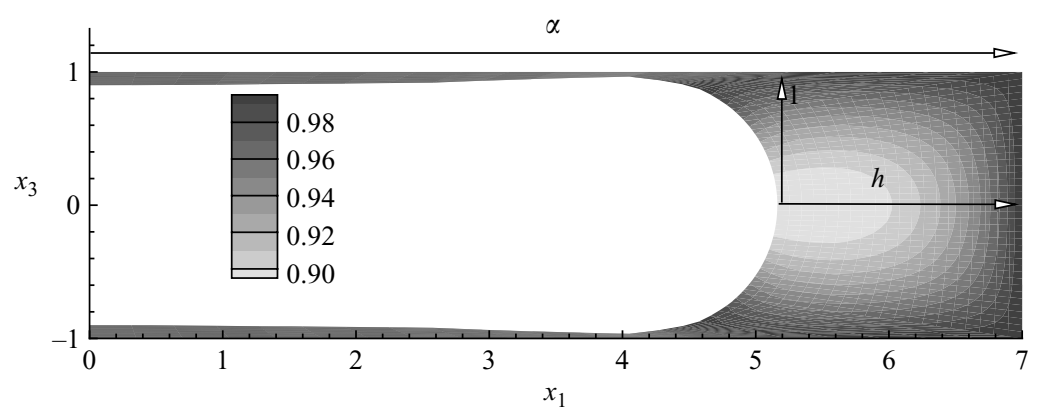

FIGURE 13. Cross-section of the flow domain at a distance $1.5 \alpha$ behind the tip for $C a=0.042, B o=0$ and aspect ratio $\alpha=7$. The grey scale represents the axial velocity.

the simulations at $B o=0$, we find $a_{1}=0.09 \pm 0.01$ and $a_{2}=0.019 \pm 0.001$, providing further evidence that the effective scaling is a good approximation when $B o \leqslant 1$. The separation between the curves increases with the Bond number, however, indicating that the physics underlying the scaling no longer applies for large $\mathrm{Bo}$. The most likely explanation for the increased error is the dramatic change in the equilibrium configurations of the finger when $B o>1$.

\subsection{Interpretation}

The wet fraction is a measure of the cross-sectional area occupied by the fluid far behind the finger tip and, in the absence of gravity, there are only three possible interface shapes in this region: (i) four quarter-circles, (ii) two semi-circles or (iii) a complete circle, see $\S 3.1$, in particular figure 5. We believe that the observed scaling of the wet fraction in rectangular tubes expresses a relationship between situations in which the interface approaches case (ii) far behind the finger tip.

An alternative scaling has been observed in case (iii), a configuration that is only possible at high capillary numbers in tubes of low aspect ratio $(1 \leqslant \alpha \lesssim 2)$. In these cases, the wet fraction appears to be independent of the aspect ratio, as predicted by Hazel \& Heil (2002) and confirmed experimentally by de Lózar et al. (2007) for $C a>0.01$.

In contrast, case (ii) is possible over a wide range of aspect ratios and capillary numbers and we believe it is this configuration that underlies the empirical scaling of de Lózar et al. (2007). Simulation results indicate that the transverse curvature (curvature in the $\left(x_{1}, x_{3}\right)$-plane) remains approximately unchanged along the finger and has a (dimensionless) value of 1 , whereas the (dimensionless) lateral curvature (curvature in the $\left(x_{1}, x_{2}\right)$-plane) is non-zero at the finger tip but becomes zero once the system is independent of the axial coordinate. Furthermore, the simulations indicate that the majority of the change in lateral curvature occurs within a short distance of the finger tip on an axial length scale of $\alpha$. We approximate the change in lateral curvature by assuming that the tip's cross-section in the plane $x_{3}=0$ is a semi-circle of radius $(\alpha-h)$, where $h$ is the lateral film thickness behind the finger tip, see figure 13. Thus the lateral curvature near the finger tip is approximately $1 /(\alpha-h)$ and we assume that it approaches zero in an adjustment region of length $\alpha$.

After the rapid changes near the tip, variations in the axial direction are assumed to be small and a standard long-wavelength approximation in the adjustment region 
leads to the governing equations

$$
C a\left(\frac{\partial^{2} u_{2}}{\partial x_{1}^{2}}+\frac{\partial^{2} u_{2}}{\partial x_{3}^{2}}\right)=\frac{\partial p_{C a}}{\partial x_{2}}, \quad \frac{\partial p_{C a}}{\partial x_{1}}=\frac{\partial p_{C a}}{\partial x_{3}}=0,
$$

where $p_{C a}=p^{*} /(\sigma / b)$, is the fluid pressure on the capillary scale. The fluid pressure is constant over each cross-section and the axial pressure gradient is approximated by the change in lateral curvature over the adjustment region

$$
C a\left(\frac{\partial^{2} u_{2}}{\partial x_{1}^{2}}+\frac{\partial^{2} u_{2}}{\partial x_{3}^{2}}\right) \sim \frac{1}{\alpha(\alpha-h)} .
$$

The fluid in the thin films above and below the air finger reaches the velocity of the wall in very short axial distances and so the viscous term can be approximated by variations in axial velocity only at the side of the finger, see figure 13 . The axial velocity is constant on the tube walls and reaches its minimum value near the air finger in the plane $x_{3}=0$, so we approximate

$$
\left(\frac{\partial^{2} u_{2}}{\partial x_{1}^{2}}+\frac{\partial^{2} u_{2}}{\partial x_{3}^{2}}\right) \propto\left(\frac{1}{h^{2}}+\frac{1}{1^{2}}\right) .
$$

The wet fraction in the equilibrium configuration is approximated by $h / \alpha$, see figure 13, and so if we wish to relate configurations in which the wet fractions are the same, we must choose $h \propto \alpha$ and if $h \approx K \alpha$, then the approximations (5.1) and (5.2) give

$$
C a\left(1+\frac{1}{h^{2}}\right) \sim \frac{1}{\alpha(\alpha-h)} \sim \frac{1}{\alpha^{2}(1-K)} \Rightarrow C a\left(K^{2} \alpha^{2}+1\right) \sim \frac{K^{2}}{(1-K)},
$$

suggesting the putative scaling parameter

$$
C a\left(1+K^{2} \alpha^{2}\right) \text {. }
$$

The two components of the viscous term will balance at a transitional aspect ratio, $\alpha_{t}$, when $h=1 \approx K \alpha_{t}$, so $K \approx 1 / \alpha_{t}$, in which case our scaling becomes

$$
C a\left(1+\frac{\alpha^{2}}{\alpha_{t}^{2}}\right)
$$

which differs from the previous empirical scaling by the disappearance of the linear term. On performing a least-squares fit to find $\alpha_{t}$ the collapse of the experimental data is comparable to that of the three-term relationship proposed by de Lózar et al. (2007), see figure 14.

The breakdown of the scaling at low $C a$ is a consequence of the fact that the interface approaches case (i) and the change in curvature can no longer be estimated from the finger width. The behaviour in this regime can be approximated by the lubrication theory approach of Wong et al. $(1995 a, b)$, in which case the functional dependence on $\alpha$ follows from the static wet fraction. For increasing Bond numbers, the widths of the equilibrium configurations increase at fixed $\mathrm{Ca}$, leading to a change in the transitional aspect ratio and consequent increase in the error of the scaling.

\section{Conclusions}

In this paper we have investigated the propagation of long bubbles into tubes of rectangular cross-sections of moderate aspect ratio $(1 \leqslant \alpha \leqslant 8)$, including the effects of 

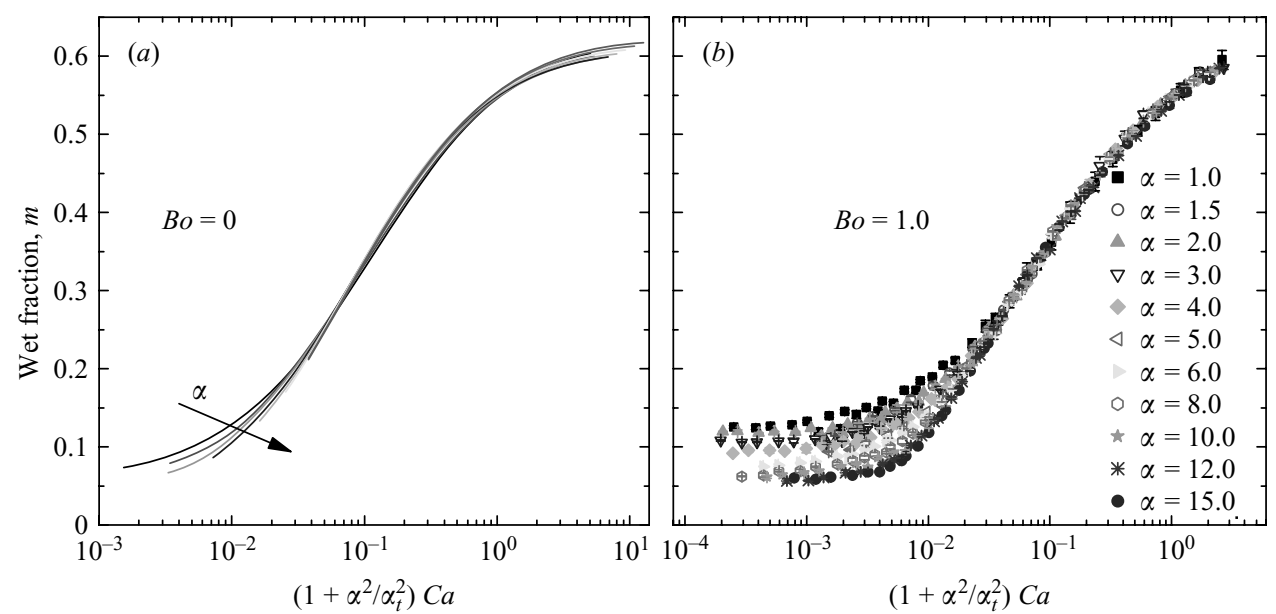

FIgURE 14. Wet fraction in $(a)$ the simulations at $B o=0$ and $(b)$ in the experiments of de Lózar et al. (2007) as a function of the modified capillary number $C a\left(1+\alpha^{2} / \alpha_{t}^{2}\right)$. Minimizing the separation of the curves in $(a)$ yields $\alpha_{t}=6.4 \pm 0.1$. The same $\alpha_{t}$ was also used in $(b)$. Note that the Bond number in the experiments $(B o=1.0)$ differs from the simulations $(B o=0)$.

transverse gravity on the flow. The predictions of bulk flow properties are in excellent agreement with our previous experiments (de Lózar et al. 2007) demonstrating the accuracy of the method.

In general, at a fixed capillary number the relative finger width and pressure drop across the finger tip decrease with increasing aspect ratio. For sufficiently large $\mathrm{Ca}$, the fraction of the cross-section occupied by the fluid after passage of the air finger increases with aspect ratio, but at low $\mathrm{Ca}$, this so-called wet fraction decreases with increasing aspect ratio. The pressure drop changes little between $\alpha=7$ and $\alpha=8$ and for these aspect ratios the relative finger width depends only on the SaffmanTaylor parameter $1 / B=12 \alpha^{2} C a$, suggesting that the system is approximately twodimensional for $\alpha \geqslant 8$. The main influence of a transverse gravitational body force is to cause an increase in pressure drop, finger width and wet fraction at fixed capillary number and aspect ratio. A dramatic increase in these quantities is observed from $B o=1.0$ to $B o=2.5$, as a consequence of the fact that at $B o>1$ it is not possible to find equilibrium interfaces that touch the bottom wall of the tube and therefore the finger lifts, as predicted by Jensen et al. (1987). The increase in finger width is driven by the enhanced draining flows from the film above the finger to that below it, causing the buoyant finger to be 'squashed' up towards the upper wall of the tube, which also results in an increase in the wet fraction.

The topology of the flow in the high- $\alpha$ tubes presents a wider variety of states than in the low- $\alpha$ ones. For $\alpha \geqslant 3$ a new flow regime occurs at intermediate capillary numbers, in which a local counterflow develops ahead of the finger tip, shielding it from contaminants. We also find that the capillary number above which the centreline flow velocity far from the tip is always slower than the bubble, $C a_{c}$, follows the simple empirical law: $C a_{c}^{2 / 3} \alpha=1.21$ for $\alpha \gtrsim 2$.

Finally, we investigated the influence of gravity on the empirical scaling of de Lózar et al. (2007) which indicates that the wet fraction depends on a modified capillary number $\widehat{C a}=C a\left[1+a_{1}(\alpha-1)+a_{2}(\alpha-1)^{2}\right]$ for all aspect ratios and for $\widehat{C a}>0.035$. The simulations confirm that the scaling is not exact, but that the errors in predictions 
using $\widehat{C a}$ are smaller than the typical experimental error $( \pm 0.01)$. The empirical scaling is applicable for $B o \leqslant 1$, but loses accuracy as the Bond number increases and the interface configurations far behind the bubble tip lift off the bottom wall of the tube. We suggest that the scaling is a consequence of a class of interface configurations in which the curvature is set by the depth of the tube. These configurations occur for higher capillary numbers over all aspect ratios $\alpha \gtrsim 2$. Simple arguments suggest that the wet fraction remains constant if the finger width scales with the aspect ratio, in which case the modified capillary number would not be expected to contain any linear terms in $\alpha$. Using a least-squares fit to find the single constant multiplying the quadratic term leads to errors comparable to those of de Lózar et al. (2007) and predicts a transitional aspect ratio of $\alpha_{t} \approx 6.4$ at which the contributions to the viscous forces in an adjustment region behind the finger tip are approximately equal in both transverse directions over a wide range of capillary numbers.

Finally, we have achieved the first quantitative agreement between direct threedimensional simulations (for $\alpha=7-8$ ) and the two-dimensional model of McLean \& Saffman (1981). Our results suggest that the two-dimensional model can accurately describe finger propagation in moderate-aspect-ratio tubes, where the values of $1 / B$ required for steady Saffman-Taylor finger propagation can be realized with relatively large values of $C a$. For small values of $1 / B$, we would expect a divergence of the results of McLean \& Saffman (1981) from the three-dimensional simulations, because the two-dimensional model requires a thin-film correction at low $C a$ (Tabeling \& Libchaber 1986).

This work was funded by EPSRC grant EP/D002214/1 (A. L.) and an EPSRC 'Advanced Research Fellowship' (A.J.). The authors would like to acknowledge the helpful comments from the referees and the editor that helped to improve an earlier version of this manuscript. In addition, A. L. H. would like to thank Dr Linda Drath and Mrs Judith Hazel for their contributions to all his JFM papers and to dedicate this work to them.

\section{Appendix. A special case: the free rise of a bubble at high $B o$}

By changing the orientation of the vector $\boldsymbol{k}$ in equation $(2.1)$ so that $\boldsymbol{k}=(0,1,0)$, we may also study the effects of a gravitational body force along the axis of the tube. As further validation of our code, we have performed simulations of the 'free' rise of air fingers in rectangular tubes, studied experimentally by Clanet et al. (2004). In this case, the base is removed from a tube that is completely filled with fluid and an air finger propagates upwards. In a stationary frame of reference the net axial flow rate will be zero, but in our moving frame the required flow rate is equal to the cross-sectional area of the tube, $Q_{c}=4 \alpha$. The velocity of the finger is fixed by the Bond number and aspect ratio and the capillary number is an unknown determined by requiring the flow rate to be $Q_{c}$.

Following Clanet et al. (2004), we define a dimensionless finger velocity based on the balance between viscous and buoyancy forces, $\mathscr{U}_{F}=C a /(\alpha B o)=\mu U_{F} /(\rho g w b) \propto$ $\mu U /(\rho g S)$, where $S$ is the surface area of the tube's cross-section. In figure 15 we plot the non-dimensional finger velocity as a function of the aspect ratio for $B o=5,22,42$ and 75. At the highest Bond numbers the curves collapse because the effects of surface tension on the bubble velocity become negligible. As the Bond number decreases the increasing importance of surface tension reduces the finger velocity at a given aspect ratio. Our results compare well with the experiments of Clanet et al., performed at 


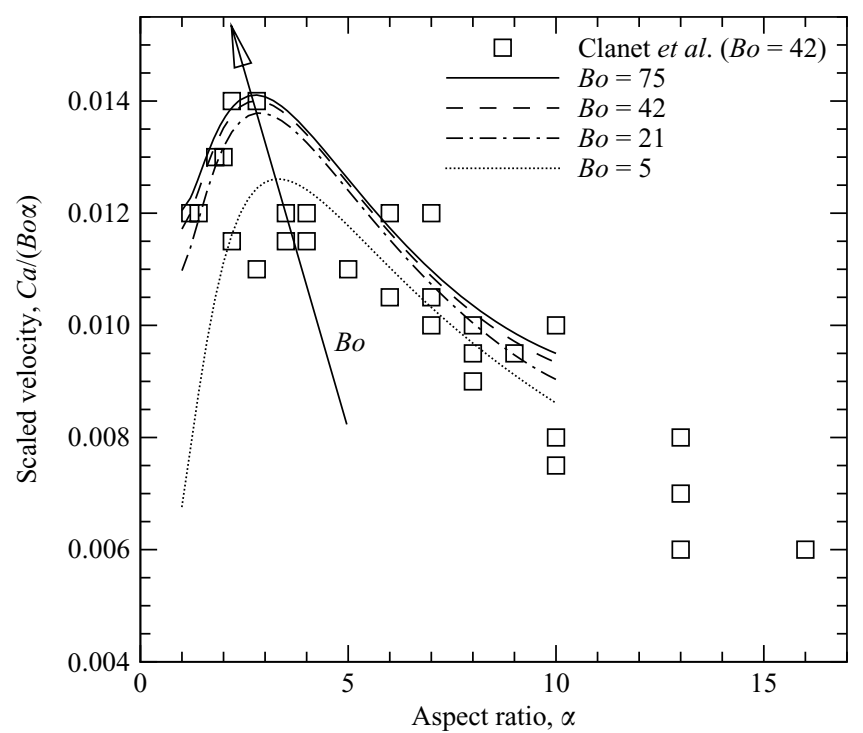

FIGURE 15. Dimensionless velocity of a 'freely' rising bubble as a function of the aspect ratio. The solid lines show the simulated results for $B o=5,22,42,75$ and the squares the experimental results of Clanet et al. (2004) for $B o=42$.

$B o=42$, shown as squares in figure 15. We note, however, that while Clanet et al. considered $\mathscr{U}_{F}$ to be a constant for low $\alpha$, we observe a well-defined maximum at $\alpha=2.8$.

\section{REFERENCES}

Ajaev, V. S. \& Homsy, G. M. 2006 Modeling shapes and dynamics of confined bubbles. Annu. Rev. Fluid Mech. 38, 277.

Baroud, C. N. \& Willaime, H. 2004 Multiphase flows in microfluidics. Compt. Rendus Phys. 7, 547.

Bretherton, F. P. 1961 The motion of long bubbles in tubes. J. Fluid Mech. 10, 166.

Clanet, C., Héraud, P. \& Searby, G. 2004 On the motion of bubbles in vertical tubes of arbitrary cross-sections: some complements to the Dumitrescu-Taylor problem. J. Fluid Mech. 519, 359.

DufF, I. S. \& ScotT, J. A. 1996 The design of a new frontal code for solving sparse, unsymmetric linear systems. ACM Trans. Math. Software 22, 30-45.

Giavedoni, M. D. \& SAita, F. A. 1997 The axisymmetric and plane cases of a gas phase steadily displacing a Newtonian liquid-A simultaneous solution of the governing equations. Phys. Fluids 9, 2420.

Gresho, P. M. \& SAni, R. L. 2000 Incompressible Flow and the Finite Element Method. Volume Two: Isothermal Laminar Flow. John Wiley \& Sons Ltd.

Hazel, A. L. \& Heil, M. 2002 The steady propagation of a semi-infinite bubble into a tube of elliptical or rectangular cross-section. J. Fluid Mech. 470, 91.

Heil, M. \& Hazel, A. L. 2006 oomph-lib-An Object-Oriented Multi-Physics Finite-Element Library. In Fluid-Structure Interaction (ed. M. Schafer \& H.-J. Bungartz), p. 19. Springer.

Homsy, G. M. 1987 Viscous fingering in porous media. Annu. Rev. Fluid Mech. 19, 271.

Jensen, M. H., Libchaber, A., Pelcé, P. \& Zocchi, G. 1987 Effect of gravity on Saffman-Taylor meniscus: Theory and experiment. Phys. Rev. A 35, 2221.

Kistler, S. F. \& SCRIVEn, L. E. 1983 Coating flows. In Computational Analysis of Polymer Processing (ed. J. R. A. Pearson \& S. M. Richardson), p. 243. Applied Science Publishers.

Kolb, W. B. \& Cerro, R. L. 1991 Coating the inside of a capillary of square cross section. Chem. Engng Sci. 46, 2181. 
Landau, L. D. \& Levich, V. G. 1942 Dragging of a liquid by a moving plate. Acta Physiocochimica URSS 17, 42.

Lister, J. R., Morrison, N. F. \& Rallison, J. M. 2006 Sedimentation of a two-dimensional drop towards a rigid horizontal plane. J. Fluid Mech. 552, 345.

de Lózar, A., Hazel, A. L. \& Juel, A. 2007 Scaling properties of coating flows in rectangular channels. Phys. Rev. Lett. 99, 234501.

McLean, J. W. \& Saffman, P. G. 1981 The effect of surface tension on the shape of fingers in a Hele-Shaw cell. J. Fluid Mech. 102, 455.

Moore, M. G., Juel, A., Burgess, J. M., McCormick, W. D. \& Swinney, H. L. 2002 Fluctuations in viscous fingering. Phys. Rev. E 65, 030601(R).

Park, C. W. \& Homsy, G. M. 1984 Two-phase displacement in Hele-Shaw cells: theory. J. Fluid Mech. 139, 291.

Ratulowski, J. \& Chang, H.-C. 1989 Transport of gas bubbles in capillaries. Phys. Fluids A 1, $1642-1655$.

Reinelt, D. A. 1987 Interface conditions for two-phase displacement in Hele-Shaw cells. J. Fluid Mech. 183, 219.

Reinelt, D. A. \& Saffmann, P. G. 1985 The penetration of a finger into a viscous fluid in a channel and tube. SIAM J. Sci. Stat. Comput. 6, 542.

RUSCHAK, K. J. 1980 A method for incorporating free boundaries with surface tension in finite element fluid-flow simulators. Intl J. Num. Meth. Engng 15, 639.

SAfFman, P. G. \& TAYLOR, G. I. 1958 The penetration of a fluid into a porous medium or Hele-Shaw cell containing a more viscous liquid. Proc. R. Soc. Lond. A 245, 312.

Tabeling, P. \& Libchaber, A. 1986 Film draining and the Saffman-Taylor problem. Phys. Rev. A 33, 794.

Tabeling, P., Zocchi, G. \& Libchaber, A. 1987 An experimental study of the Saffman-Taylor instability. J. Fluid Mech. 177, 67.

TAYLOR, G. I. 1961 Deposition of viscous fluid on the wall of a tube. J. Fluid Mech. 10, 161.

Whitesides, G. M. \& Stroock, A. D. 2001 Flexible methods for microfluidics. Physics Today 54 (6), 42.

Wong, H., Radke, C. J. \& Morris, S. 1995a The motion of long bubbles in polygonal capillaries. part 1. thin films. J. Fluid Mech. 292, 71.

Wong, H., RadKe, C. J. \& Morris, S. $1995 b$ The motion of long bubbles in polygonal capillaries. Part 2. Drag, fluid pressure and fluid flow. J. Fluid Mech. 292, 95. 